\title{
Synchronization and coordination of sequences in two neural ensembles
}

\author{
Antoine Venaille, Pablo Varona, ${ }^{*}$ and Mikhail I. Rabinovich ${ }^{\dagger}$ \\ Institute for Nonlinear Science. University of California, San Diego, 9500 Gilman Dr. 0402, La Jolla, California 92093, USA
}

(Received 12 August 2004; published 21 June 2005)

\begin{abstract}
There are many types of neural networks involved in the sequential motor behavior of animals. For high species, the control and coordination of the network dynamics is a function of the higher levels of the central nervous system, in particular the cerebellum. However, in many cases, especially for invertebrates, such coordination is the result of direct synaptic connections between small circuits. We show here that even the chaotic sequential activity of small model networks can be coordinated by electrotonic synapses connecting one or several pairs of neurons that belong to two different networks. As an example, we analyzed the coordination and synchronization of the sequential activity of two statocyst model networks of the marine mollusk Clione. The statocysts are gravity sensory organs that play a key role in postural control of the animal and the generation of a complex hunting motor program. Each statocyst network was modeled by a small ensemble of neurons with Lotka-Volterra type dynamics and nonsymmetric inhibitory interactions. We studied how two such networks were synchronized by electrical coupling in the presence of an external signal which lead to winnerless competition among the neurons. We found that as a function of the number and the strength of connections between the two networks, it is possible to coordinate and synchronize the sequences that each network generates with its own chaotic dynamics. In spite of the chaoticity, the coordination of the signals is established through an activation sequence lock for those neurons that are active at a particular instant of time.
\end{abstract}

DOI: 10.1103/PhysRevE.71.061909

PACS number(s): 87.19.La, 05.45.Xt, 87.18.Sn

\section{INTRODUCTION}

The generation of sequences is a main function of the neural networks that are responsible for animal motor behavior. For rhythmic activity like breathing, heart beating, or running, neural networks called central pattern generators (CPGs) produce periodic or nearly periodic activity sequences or spatiotemporal patterns. Different motor neurons in such patterns are active in different phases of the rhythmic cycle. In more complex cases such as the hunting swimming of the marine mollusk Clione or the singing of songbirds, the motor sequence required can be nonperiodic, chaotic, or transient depending on the stimuli $[1,2]$. There are several different networks including CPGs that are usually involved in the organization of specific sequential motor activity. The problem of synchronization and coordination of sequential activities of neurons from different complex networks is crucially important for a well organized motor behavior. In this paper we investigate the simplest case, i.e., the coordination and synchronization of sequences in two small networks. We analyze the sequence coordination and synchronization depending on the type and strength of the coupling between the networks.

The inhibitory networks that we study here are built with neurons that compete to be active. None of the cells can dominate the others for a long time, i.e., there is a sequential switching activity in the neural ensembles. This process is

\footnotetext{
*Also at Grupo de Neurocomputación Biológica (GNB), Dpto. de Ingeniería Informática, Universidad Autónoma de Madrid, 28049 Madrid, Spain.

†Electronic address: mrabinovich@ucsd.edu; URL: http:// inls.ucsd.edu
}

referred to as winnerless competition (WLC). While the concept of WLC has been experimentally known for a long time [3], its application to neural processing within dynamical systems is more recent $[4,5]$. In each experiment involving WLC there is a sequential switching of different patterns in which none of them prevails over the others. This process can be described by a generalized Lotka-Volterra model:

$$
\dot{a}_{i}=a_{i}\left[1-\left(a_{i}+\sum_{i \neq j}^{N} \rho_{i j} a_{j}\right)\right]+G_{i},
$$

where $a_{i} \geqslant 0, \rho_{i j} \geqslant 0$, and the function $G_{i}$ represents the effect of stimulus-dependent interactions between neurons. For $N$ $=3$ and $G_{i}=0$, this is a well known kinetic equation in population biology as it was first introduced to describe the population evolution of fish species $i$ characterized by the number $a_{i}$ of individuals [6]. The impact on the growth rate $a_{i}$ of the $i$ th species by the presence of the $j$ th species is determined by the competition coefficient $\rho_{i j}$. One essential feature to trigger WLC behavior in the population evolution is the nonsymmetry of coefficients $\rho_{i j} \neq \rho_{j i}$. The mathematical image of WLC is a stable heteroclinic sequence in the phase space: saddle points connected with one-dimensional separatrices. This simple model can describe networks with inhibitory connections that one can meet in economy [7], in mode competition in physics [8], or in neural systems [4,9]. At first view the WLC heteroclinic sequence may seem very exotic and structurally unstable. However, even for dissipative systems like Eq. (1) with $G_{i}=0$, which have an invariant subspace [a $(N-1)$-dimensional simplex in our case [10]], the WLC dynamics can be robust and persistent under arbitrary perturbations (see also Ref. [11] for the same phenomena in a different system). It is also possible to prove that the system (1) with $G_{i}$ a linear function of $a_{i}$ has a surface $S_{0}$ that 

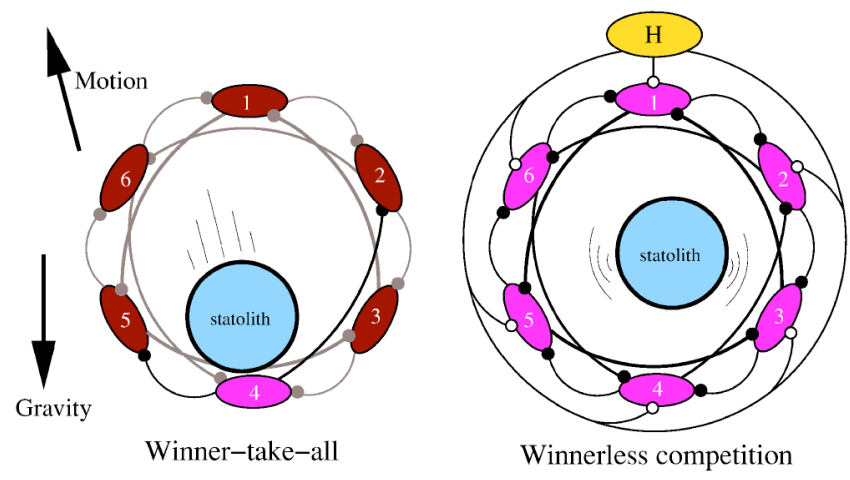

FIG. 1. (Color online) Schematic representation of the dual role of a single statocyst with six receptor neurons. During normal swimming, a stonelike structure, the statolith hits the mechanoreceptor neurons that react to this excitation. In Clione's hunting behavior, the statocyst receptors receive an additional excitation from the cerebral hunting neuron $(H)$ that generates a winnerless competition among them.

covers part of the phase space including the origin and all fixed points in which all trajectories intersect, i.e., $\left.\dot{a}_{i}\right|_{S_{0}}<0$, $i=1, \ldots, N$. Thus such a system has no trajectories that escape to infinity.

The WLC principle has made possible the interpretation, for example, of the hunting search behavior of Clione, a small blind marine mollusk $[1,9]$. This is a planktonic animal negatively buoyant that has to maintain a continuous motor activity in order to keep its preferred head-up orientation. The motor activity is controlled by the wing central pattern generator and the tail motoneurons that use the signals from its gravity sensory organs, the statocysts [13]. A six receptor neural network model with synaptic inhibitions has been built to describe a single statocyst [9] (see Fig. 1). This is a small sphere in which the statolith, a stonelike structure, moves according to the gravitational field. The statolith excites the neuroreceptors by pressing them. When excited, the receptors send signals to the neural systems responsible for wing beating and tail orientation. The statocysts have a dual role [1]. During normal swimming only the neurons that are excited by the statolith are active which leads to a winnertake-all mode as a result of inhibitory connections in the network. However, during hunting search behavior, a cerebral neuron excites each neuron of the statocyst (see right panel in Fig. 1). This triggers WLC between all statocyst neurons whose signals participate in the generation of a complex motion that the animal uses to scan the space until it finds its prey [1]. The image of the chaotic dynamics of the statocyst model in this behavioral mode is a strange attractor and the heteroclinic loops become unstable. We have shown [9] that for $N=6$ the observed dynamical chaos is characterized by two positive Lyapunov exponents whose magnitudes do not change when increasing the accuracy of the computation. That means that the origin of chaos is not related to numerical noise. We also showed that chaotic activity also exists when the statocyst network is modeled with conductance-based models [12].

The ability of a single statocyst to generate a chaotic activity resulting in randomlike movement has been experi- mentally and theoretically well understood $[1,5,9]$. The statocyst model using Lotka-Volterra type neurons has made predictions about the switching dynamics in this network that have been tested experimentally [1]. However, the animal has two statocysts electrically coupled, and the effect of their interaction is less known. This could not only be interesting from the biological point of view, i.e., what physiological features of Clione behavior can be understood from the interaction of both networks, but also from a physical point of view: how the coupling between two networks is organized to generate two nonidentical chaotic sequences that, nevertheless, have features that favor their coordination.

Recent experiments have shown that both the tail motoneurons and the wing CPG receive the influence of the statocyst signals [1]. The actual motor movements of the wings and the tail are very different. A possible explanation for this strong influence is the role of the statocyst signal in coordinating wing and tail activity. Wing beating frequency changes constantly during hunting behavior and the tail bends into different directions in a longer time scale. However, wing and tail activity have to be coordinated to produce an effective hunting search. The activity of the statocyst network seems to participate not only in the hunting motor program, but also in the coordination of the tail and the wing motor activity.

The synchronization of low dimensional chaotic systems has been widely studied [14-17]. In this paper we consider the coordination and synchronization of chaotic sequences in two six-dimensional networks that represent a sensory organ with dual function. Our results show that while producing the highly irregular signals required for their functional role during hunting, the two networks can also display additional features suitable for the coordination of neural activity.

\section{MODEL}

We have built two neural ensembles $A$ and $B$. Each of them represents the network of neuroreceptors in a statocyst. In our model each network is composed of six neurons. Physiological recordings [13] have lead to establish some rules to build the statocyst network, and in particular to choose the inhibition matrix: $30 \%$ of the neuron pairs are connected with inhibitory connections. Here we assume that each neuron receives and sends two inhibitory signals when activated (see Fig. 2). Moreover, each neuron $a_{i}$ or $b_{i}$ of network $A$ or $B$, respectively, receives a signal $H_{i}^{A}$ or $H_{i}^{B}$ from the cerebral neurons that trigger the hunting search behavior.

In our study we connected neurons $a_{i}$ and $b_{i}$ belonging to networks $A$ and $B$, respectively, through electrical coupling (see Fig. 2); the conductance of the coupling $g_{i}$ determines the strength of the connection. During hunting, the role of the statolith is considered negligible and thus we do not discuss here its effect on the dynamics of the networks.

The dynamics of the two statocyst networks is described with a set of 12 ordinary differential equations based on a generalized Lotka-Volterra type dynamics:

$$
\dot{a}_{i}=a_{i}\left(1-\sum_{j=1}^{N} \rho_{i j}^{A} a_{j}+H_{i}^{A}\right)-g_{i}\left(a_{i}-b_{i}\right),
$$




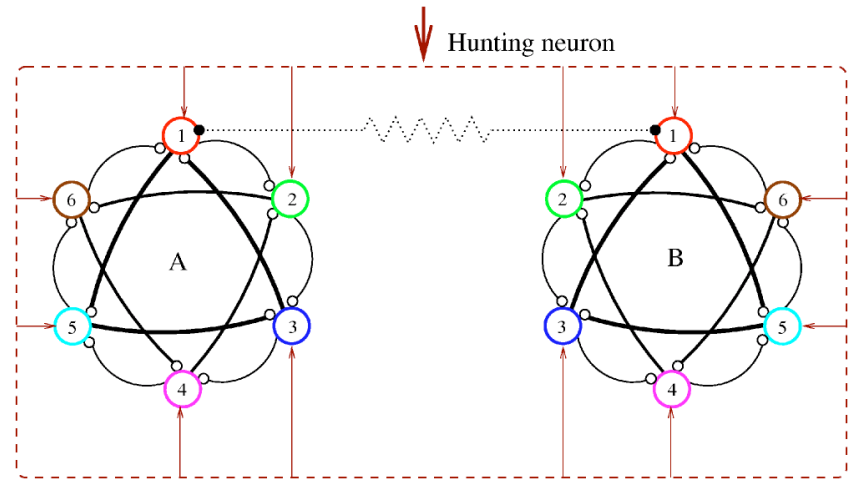

FIG. 2. (Color online) Network configuration used in our study. Resistor represents the electrical coupling between the two networks. Inhibitory connections within each ensemble are represented by white dots. Thicker traces mean stronger connections. The excitatory action of the hunting neuron on all the cells is represented by the arrows. In our study we change the number of connections and the strength of the coupling between these two networks.

$$
\dot{b}_{i}=b_{i}\left(1-\sum_{j=1}^{N} \rho_{i j}^{B} b_{j}+H_{i}^{B}\right)-g_{i}\left(b_{i}-a_{i}\right),
$$

where $N=6$, and $a_{i}$ and $b_{i}$ represent the spiking rate of the neurons in the network $A$ and $B$, respectively. The connection matrices $\rho^{A}$ and $\rho^{B}$ and the stimuli from the cerebral hunting neuron $\vec{H}^{A}$ and $\vec{H}^{B}$ that we will use here are

$$
\begin{aligned}
\rho^{A} & =\left(\begin{array}{cccccc}
1 & 0 & 5 & 0 & 0 & 1.5 \\
1.5 & 1 & 0 & 2 & 0 & 0 \\
0 & 1.5 & 1 & 0 & 5 & 0 \\
0 & 0 & 1.5 & 1 & 0 & 2 \\
5 & 0 & 0 & 1.5 & 1 & 0 \\
0 & 2 & 0 & 0 & 1.5 & 1
\end{array}\right) \\
\vec{H}^{A} & =\left(\begin{array}{l}
0.730 \\
0.123 \\
0.301 \\
0.203 \\
0.458 \\
0.903
\end{array}\right) \vec{H}^{B}=\left(\begin{array}{l}
0.301 \\
0.206 \\
0.458 \\
0.903 \\
0.730 \\
0.123
\end{array}\right)
\end{aligned}
$$

The inhibition matrix $\rho^{A}$ and $\rho^{B}$ are similar, except that if $\rho_{i \neq j}^{A} \neq 0$ then $\rho_{i \neq j}^{B}=\rho_{i \neq j}^{A}+0.02$. There are two biological motivations for the small differences between networks $A$ and $B$ : (i) as other sensory organs, both statocysts are not exactly identical. However, the offset in the connection matrices cannot be too high for it would not lead to WLC; (ii) different external signals (here from the hunting neurons) can arrive on the two sensory receptors. Moreover, we are interested in our study that both networks lead to similar behavior, but with different neuronal activity before the coupling to discuss later their synchronization.

\section{RESULTS}

\section{A. Independent activity}

A wide range of inhibition coefficients $\rho_{i j}$ can trigger WLC but there is often one or more neurons silent. One can see in Fig. 3 that for the values chosen here each neuron is active when there is no coupling between networks $A$ and $B$ $\left(g_{i}=0, \forall i\right)$. The calculation of the Lyapunov exponents from the vector field (2a) and (2b) yields in this case two positive exponents for each network: $\lambda_{1}^{A}=0.015, \lambda_{2}^{A}=0.004$, and $\lambda_{1}^{B}$ $=0.012, \lambda_{2}^{B}=0.002$.

Let us consider first a single network without coupling [Eqs. (2a) and (2b) with $g_{i}=0$ ]. Although a detailed analysis is possible for $N=3$ [9], this is not the case for more complex networks. When $N>3$, the system may have several different heteroclinic orbits according to the strength of inhibition. This has been demonstrated to be a powerful strategy for modeling memory [18] and other mechanisms of information processing [5]. Those considerations give theoretical support for the six neuron model: the ensemble is built with two triangular subnetworks of inhibitory neurons (see Fig. 2). The individual network dynamics of each triangle, which is weakly coupled to the other through the peripheral connections, can be understood from the three-dimensional case. The origin of the chaoticity in the six-dimensional dynamical system can be intuitively explained in the following manner: independently, each triangle has a closed heteroclinic orbit that becomes a strange attractor or a limit cycle under the action of a small perturbation. This perturbation comes here from the hunting neuron and from the inhibitory connections with the other triangle. The weak interactions of these two winnerless competitive nonlinear oscillators generate chaos over a large region of parameters.

As mentioned above, the individual statocyst dynamics is determined by the external inputs $\left(H_{i}\right)$ and by the nonsymmetrical inhibitory connections between neurons that compete against each other. In some regions of the control parameters, as the result of such competition, the output spatiotemporal pattern of the network produces a sequence of pulses generated by different neurons at different instants of time, i.e., WLC. The origin of the sequence generation can be easily explained in terms of the inhibition. Suppose that each neuron can be just in two states, i.e., silent or active. When a neuron is active, it inhibits several other neurons within the network. These silent neurons, in turn, allow the activation of the neurons that they inhibited before, and so forth. Thus it is not difficult to formulate the conditions for the connection matrix, e.g., inequalities for the values of $\rho_{i j}$ [5]. The excitation from the hunting neuron and the nonsymmetrical inhibitory connections guarantees the presence of the switching. Such sequential firing or switching can be periodic or chaotic depending on the control parameter values (connection matrix and hunting vector).

The chaotic sequences generated by the discussed networks have a very interesting specific feature. In spite of the hyperchaoticity (the corresponding strange attractor is characterized by two positive Lyapunov exponents), the order of the neural switching is invariant for those neurons that are activated in a particular time window; only the time intervals 


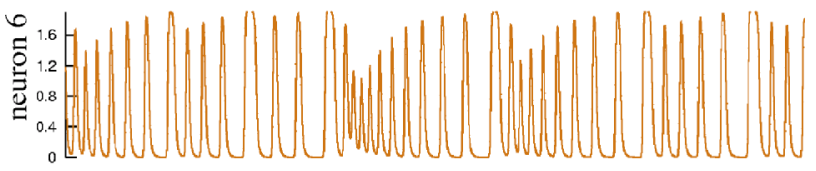

(a) 1.6
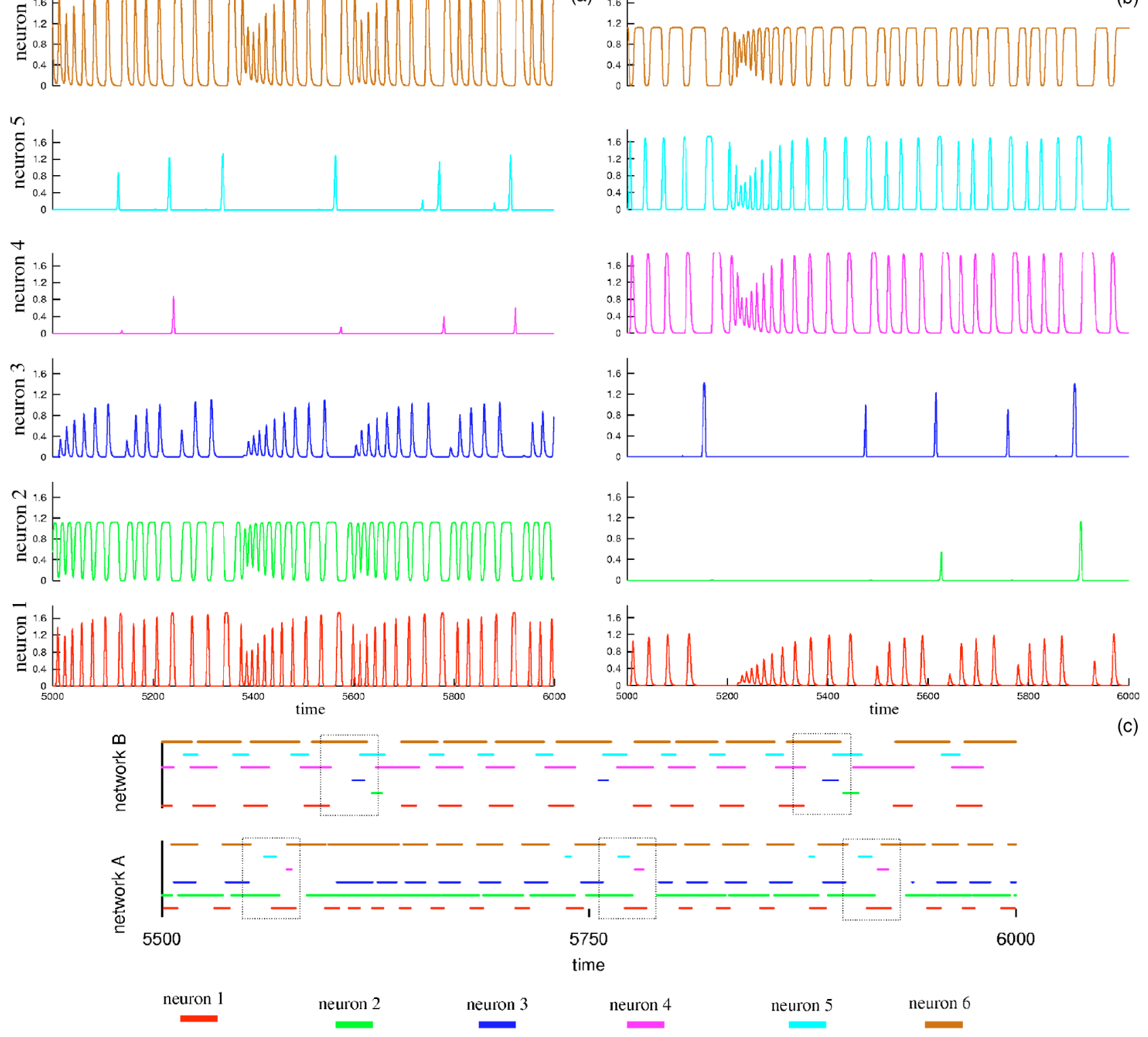

FIG. 3. (Color online) Chaotic sequential switching displayed by the activity of the neurons in network (a) and of network (b) when there is no coupling between the networks. Units are dimensionless. Panel (c) displays the time intervals in which each neuron is active ( $a_{i}$ $>0.03$ ). Each neuron is represented by a different color/gray scale. The dotted rectangles point out the activation sequence lock among the units that are active at a given time interval within each network for time windows in which all six neurons are active.

between the switching are chaotic (see panel $\mathrm{C}$ in Fig. 3). Thus activation sequence lock in this context means that the order of activation of the neurons in a short time window is the same. Note that not all the neurons need to be activated in a particular sequence. However, the order in the sequence for those that are activated is preserved.

Based on this knowledge it is reasonable to expect that, as a result of coupling between two similar but not identical networks, the corresponding output sequences can be coordinated in some sense or even synchronized. By coordination below we mean a stable regime with a fixed order of the switching in the sequences (in those neurons that are activated at a particular time window) but with uncorrelated timing. Complete synchronization means that both chaotic time series have the same order of switching and the same timing.
We can hypothesize that a richer dynamics will arise from the connection between two such networks, so that more complex signals can be generated in these sensory receptors during hunting behavior. This hypothesis will be tested in the next section.

\section{B. Coupled networks}

Electrophysiological recordings have shown that in Clione both statocysts are electrically coupled, although the degree and strength of these connections are unknown [13]. Using our model we can analyze the effect of coupling one or more receptor neurons belonging to each statocyst on the common dynamics of the two networks.

If only one neuron is connected to the corresponding cell in the other network (see Fig. 4), there is an almost complete 


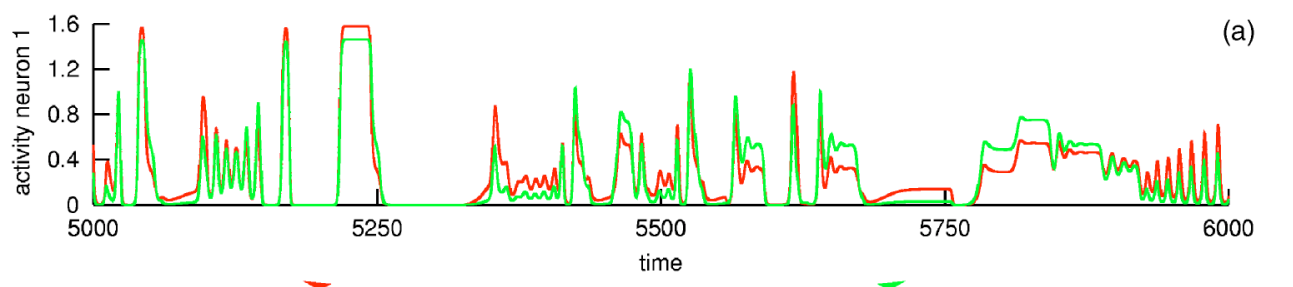

(a)
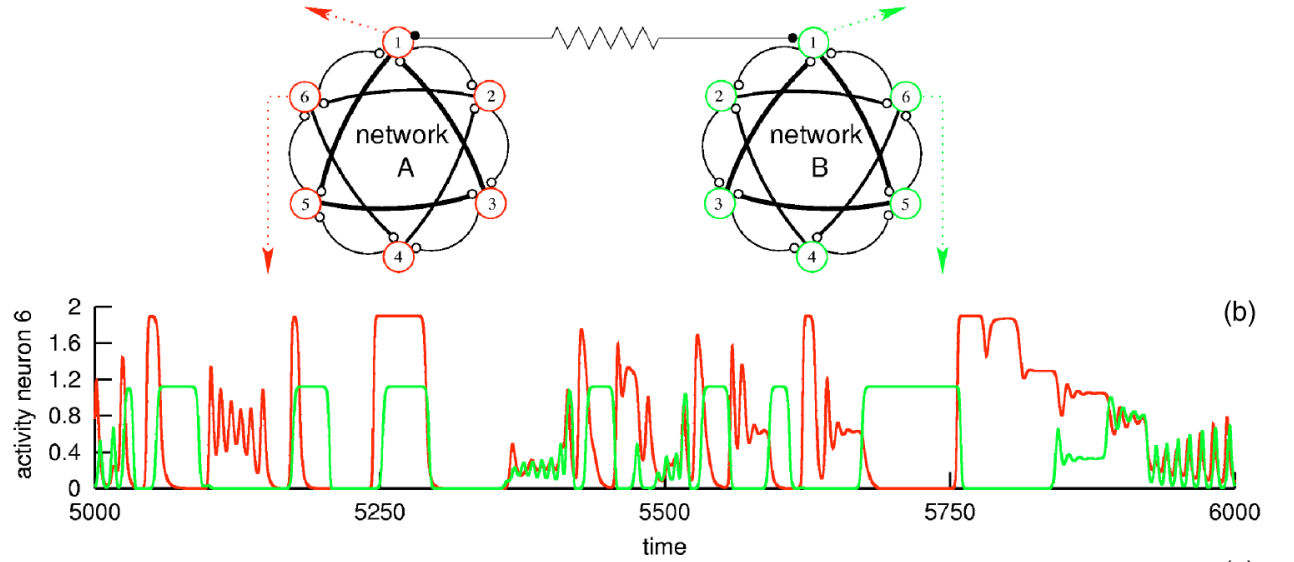

(c)

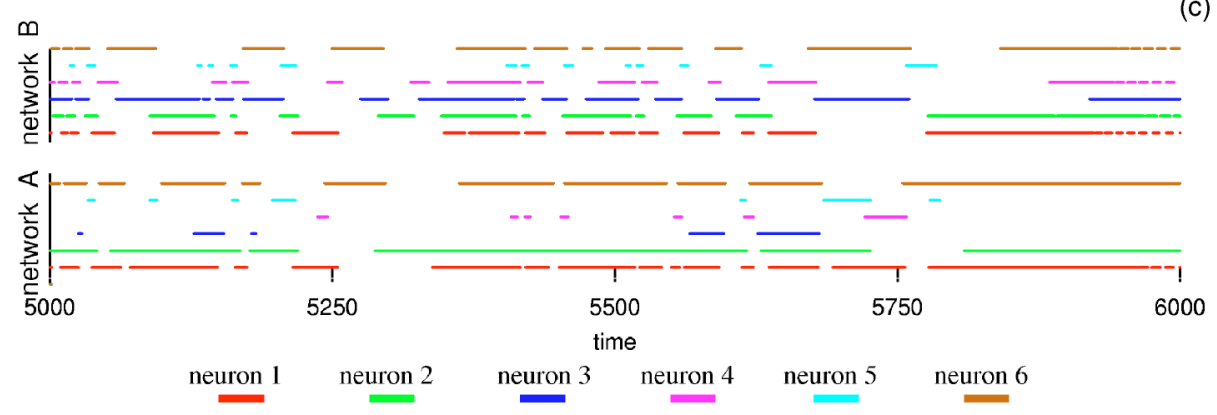

FIG. 4. (Color online) Activity of the networks with one electrotonic connection between them. The coupling vector is $g$ $=(2,0,0,0,0,0)$. Panel (a) shows the synchronized activity of neurons labeled as 1 in both networks. Panel (b) shows the activity of neurons labeled as 6. Panel (c) shows the time intervals in which each neuron is active $\left(a_{i}\right.$ $>0.03)$. Only intermittent activation sequence locks can be observed for neurons within the same network.

synchronization of the activation time of the connected neurons $\left(a_{1}\right.$ and $b_{1}$, with $\left.g_{1}=2\right)$. In panel A of Fig. 4 one can see that these two neurons fire and remain silent at the same epochs but with a slightly different amplitude. The rest of corresponding neurons in both networks have a large degree of independent behavior. As an illustration of this, panel B in Fig. 4 displays the activity of neurons $a_{6}$ and $b_{6}$ that have no direct connection. There is no synchronization in the activity of these neurons: there are several epochs in which neuron $a_{6}$ remains silent whereas neuron $b_{6}$ is active; However, transient synchronization and activation sequence locks can also be observed at some time windows when both neurons are active. Panel $\mathrm{C}$ shows that there is no continuous sequence lock between neurons of different networks except for those neurons that are directly coupled. It is also difficult to find periods with a sustained activation sequence lock for all neurons within the same network. The switching is still chaotic in both networks. In this case the calculation of the Lyapunov exponents yields that three of them are positive and the Kolmogorov-Sinai entropy $h_{K S}=\Sigma_{\lambda_{i}>0} \lambda_{i} \simeq 2$. This fact is understandable: the initial system had two separated six neuronal ensembles; it becomes a 12 neuronal ensemble when adding the electrical connection, with more combinations available for the switching activity. The connection in this case seems to diminish to a great extent the activation sequence lock present in the independent networks. However, if additional connections with a similar strength are built, for example between neurons $a_{2}$ and $b_{2}$, we observe synchronization for the neurons connected and an increased activation sequence locking for the others.

So far we have seen that it is possible to synchronize individual neurons of each network by connecting them. But is it possible to have a strong activation sequence locking between the neurons of different networks while keeping the chaoticity? As mentioned before, each of the six-neuron networks is built out of two oscillator subcircuits that consist of three neurons (two triangles) and an external loop connecting them. We have tried to synchronize the neurons of the stronger inhibitory triangle of networks $A$ and $B$ by coupling them $\left(g_{1}=g_{3}=g_{5}=0.01\right)$. Panels A and B of Fig. 5 show the activity of neurons in each of the two triangle subnetworks. Although there is no complete synchronization between the neurons of the connected triangle, we see that they both activate and deactivate at the same time (panel B). However, the activity of neurons that belong to the other triangle (without internetwork connections) are not synchronized (panel A). Panel $\mathrm{C}$ shows that in this case a high degree of activation sequence lock is present for all the neurons, even among neurons that belong to the two different networks. The calculation of the Lyapunov exponents from the vector field yields five positive exponents in this case and $h_{K S}=\Sigma_{\lambda_{i}>0} \lambda_{i}$ $\simeq 6$. The Kolmogorov-Sinai entropy is two orders of magnitude larger than in the case of the independent six neuron networks. 

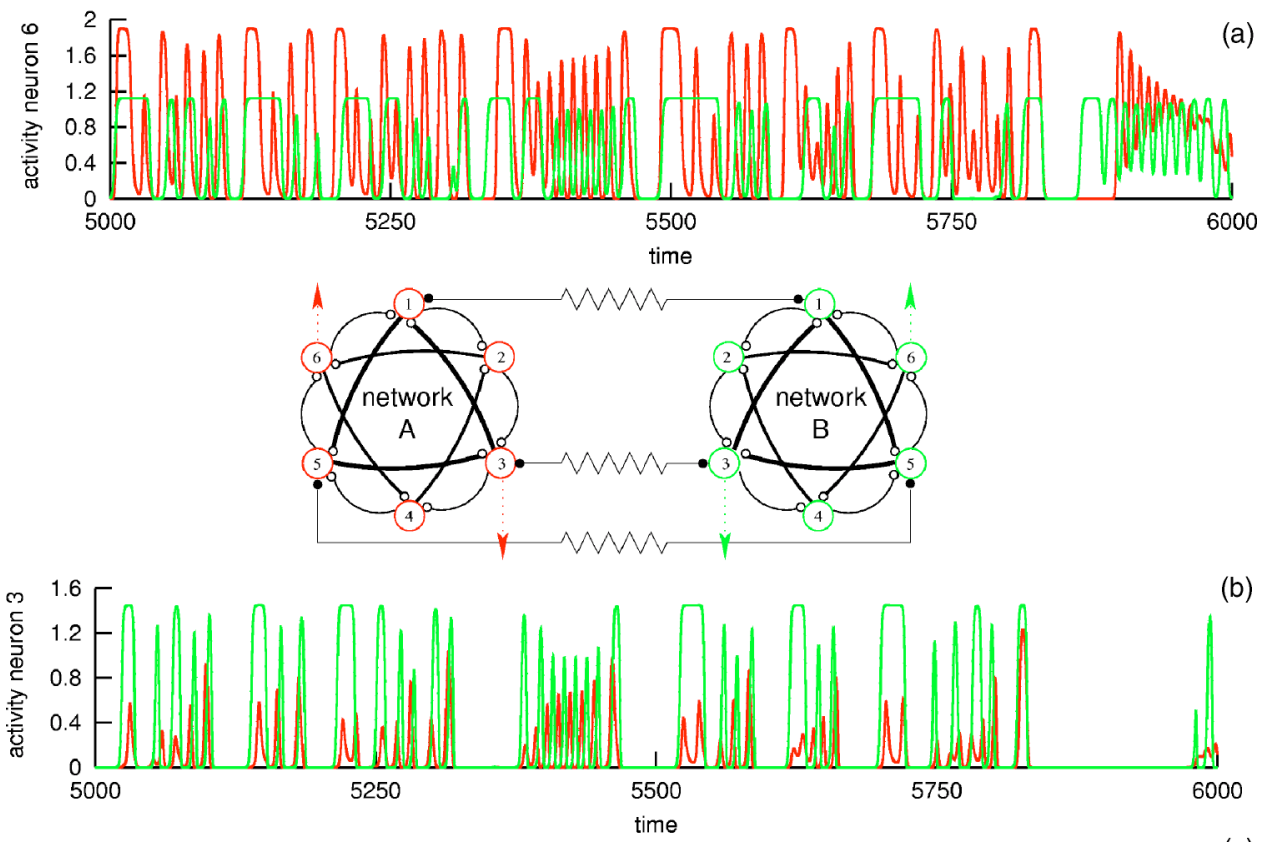

(c)

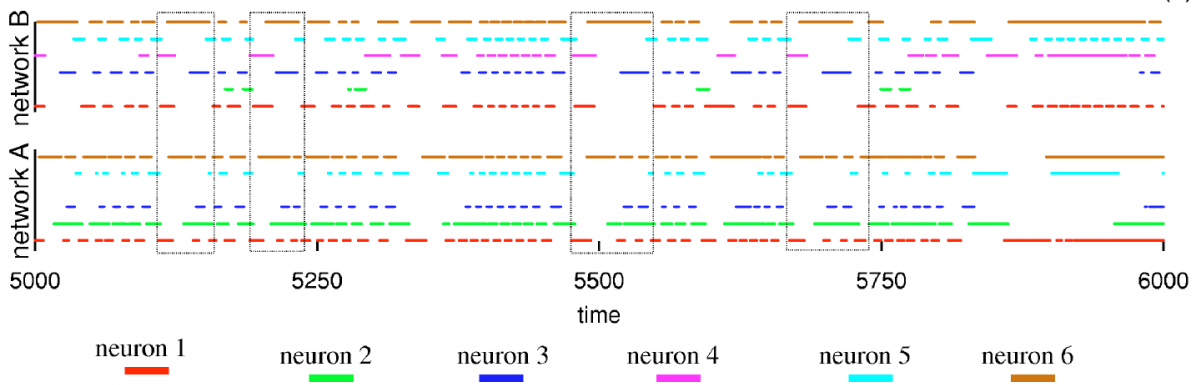

FIG. 5. (Color online) Activity of the networks with three electrotonic connections between them. The coupling vector is $g$ $=(0.01,0,0.01,0,0.01,0)$. Panel (a) shows activity of neurons labeled as 6 in both networks. Panel (b) shows the activity of neurons labeled as 3. Panel (b) shows the time intervals in which each neuron is active $\left(a_{i}>0.03\right)$. Note the activation sequence lock even among neurons of different networks (the dotted rectangles point out some examples).
One way to obtain complete synchronization for all neurons is to connect each neuron $a_{i}$ with its corresponding neuron $b_{i}$. Such a complete synchronization was not observed with less than six connections (see Fig. 6). We found that, for a one-to-one connection architecture $\left(a_{i}\right.$ to $\left.b_{i}\right)$, the two networks could display very different behaviors depending on the strength of the coupling constant $g_{i}=g, \forall i$. With small coupling $\left(g \approx 10^{-6}\right)$, the activity of each neuron is periodic with the same frequency, and both neurons $a_{i}$ and $b_{i}$ oscillate in antiphase. In this case the coupling term in Eqs. (2a) and (2b) has the same effect as an inhibitory connection which drives the system to a limit cycle. However, the system remains chaotic when the coupling conductances are lower than $g=10^{-9}$ or higher than $g=10^{-6}$, displaying in all cases the activation sequence lock.

With a strong coupling (up to $g=0.12$ ), there is complete synchronization for the chaotic activity of $a_{i}$ and $b_{i}$, with slight differences in the amplitude of the signals. In this case, there is a single positive Lyapunov exponent. However, if the coupling is too high $\left(g_{i}>0.12\right)$ the sequential switching is destroyed and the system displays no oscillations after a transient period.

The study of the two electrically coupled networks shows that there is a wide variety of rich behaviors as a function of the coupling. The two networks are able to generate chaotic signals with an activation sequence lock for several connection architectures. Even for the cases in which the sequence

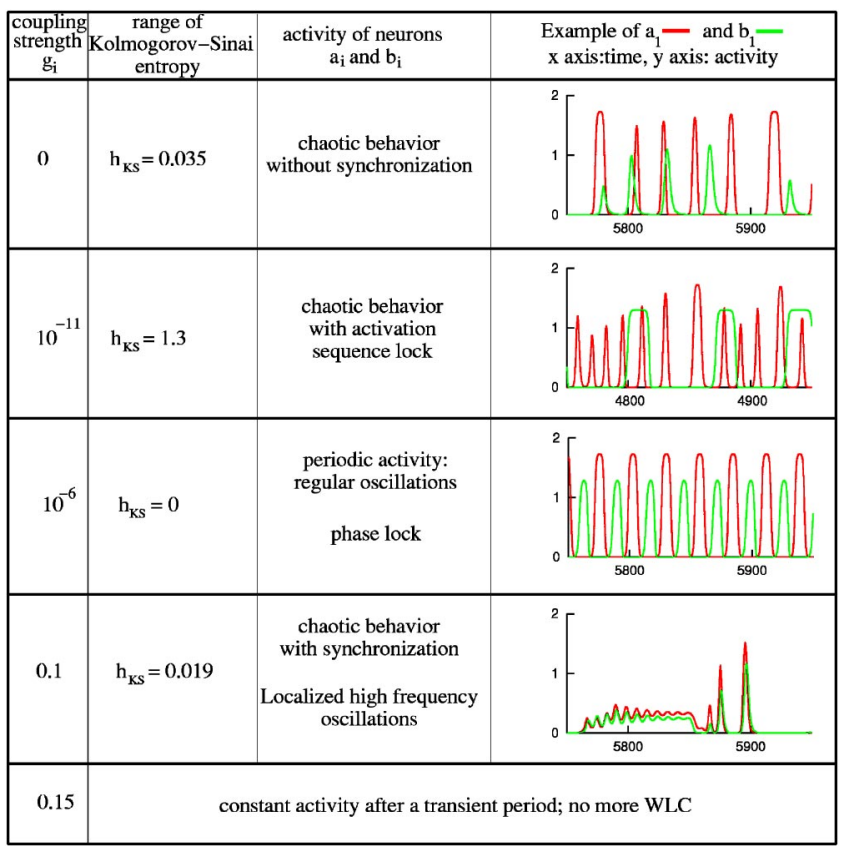

FIG. 6. (Color online) Description of the network activity when all the neurons of the two networks are electrically coupled one to one $\left(a_{i}\right.$ to $\left.b_{i}\right)$ as a function of the strength of the coupling $g_{i}$. 
lock is intermittent, this can be a desirable feature of a neural system that has to produce multiple signals to drive a complex but at the same time coordinated motion.

\section{DISCUSSION}

In this paper we have studied the coordination and synchronization between two sensory organs described with small neural ensembles. One could argue that neural networks (and mostly sensory ones) are often larger than our six neuron statocyst model. However, our approach is to look at the dynamics of small building blocks or microcircuits to understand the dynamical behavior of larger networks as in Ref. [19]. Here we have seen that two coupled statocyst networks provide richer dynamics for the sequential switching. The activation sequence locking observed in the single networks is preserved when the two ensembles are coupled with a sufficient number of connections. Furthermore, the switching can now take place among neurons of different networks. This greatly increases the richness of the sequential signals. Clione uses the signals from the statocyst to generate and coordinate two distinct motor activities during hunting: the acceleration of the wing beating and the bending of the tail in different directions. Both the acceleration of the wings and the tail bending appear to change randomly, but they are nevertheless coordinated to produce a coherent motion. In this context, the generation of a chaotic signal with activation sequence locks is an appropriate feature of the statocyst network. Sensory organs are generally receptors that gather information about the environment; then the central nervous system integrate incoming signals and adapt the response to external conditions. But for Clione's gravimetric organs things seem to work on a different way. These organs have dynamics on their own and generate complex signal that are used to generate the hunting search motor program. On that respect statocyst can actively participate in a motor behavior. WLC among the receptor neurons allow this transition between sensory activity (winner-take-all mode) and complex locomotor activity when Clione is hunting. The two statocyst networks can together create new information with features suitable for their use in driving complex but coordinated movements.

It is common to find in physiology processes that involve the synchronization between different organs [20]. For instance, the heartbeat has been known to be synchronized with ventilation in some cases [21]. But unlike hunting behavior of Clione, most physiological processes use periodic or nearly periodic oscillations. As a matter of fact neural networks often require rhythmicity for the response imposed to the living organism by the environment which is not consistent with chaotic behavior. Our analysis showed that a small interaction between the two networks leads to the regularization of the chaotic dynamics making their sequential activity purely periodic as for two coupled CPG chaotic neurons [22]. For the hunting search behavior of Clione such statocyst dynamics is not useful. On the other hand, a moderate coupling can coordinate the two network dynamics through the control of the order of the sequential switching and leave to both of them enough degrees of freedom to generate signals with high informational content. We think that these results are widely spread and nature could use this approach in other contexts. For example, the brain of birdsongs has two high vocal centers (HVCs) that generate sequences that control the rhythm and motif of the songs. The neural system of birdsongs is bilaterally organized and a good song that has a sexual meaning is the result of the coordination of the sequential dynamics of both left and right HVCs [23]. The song sequence is not random, but is not periodic nor completely predictable. We think that the coordination (not a complete synchronization) of the HVCs sequential dynamics, through the acoustic feedback, for example, is able to solve the contradiction between the richness of the song and reproducibility of the motif.

\section{ACKNOWLEDGMENTS}

We thank Dr. Allen I. Selverston for helpful comments on this manuscript. This work was supported by National Institute of Neurological Disorders and Stroke Grant No. 7R01NS-38022, National Science Foundation Grant No. EIA0130708, Fundación BBVA and Spanish MCyT Grant No. BFI2003-07276.
[1] R. Levi, P. Varona, Y. I. Arshavsky, M. I. Rabinovich, and A. I. Selverston, J. Neurophysiol. 91, 336 (2004).

[2] R. H. Hahnloser, A. A. Kozhevnikov, and M. S. Fee, Nature (London) 419, 65 (2002).

[3] F. H. Busse and K. E. Heikes, Science 208, 173 (1980).

[4] M. Rabinovich, A. Volkovskii, P. Lecanda, R. Huerta, H. D. I. Abarbanel, and G. Laurent, Phys. Rev. Lett. 87, 068102 (2001).

[5] V. S. Afraimovich, M. I. Rabinovich, and P. Varona, Int. J. Bifurcation Chaos Appl. Sci. Eng. 14, 1195 (2004).

[6] N. MacDonald, Trees and Networks in Biological Models (Wiley, New York, 1983).

[7] O. Malcai, O. Biham, P. Richmond, and S. Solomon, Phys. Rev. E 66, 031102 (2002).
[8] M. I. Rabinovich, A. B. Ezersky, and P. D. Weidman, The Dynamics of Patterns (World Scientific, Singapore, 2000).

[9] P. Varona, M. I. Rabinovich, A. I. Selverston, and Y. I. Arshavsky, Chaos 12, 672 (2002).

[10] E. C. Zeeman and M. L. Zeeman, Nonlinearity 15, 2019 (2002).

[11] P. Ashwin and J. Borrensen, eprint q-bio.NC/0409012.

[12] P. Varona, R. Levi, Y. I. Arshavsky, M. I. Rabinovich, and A. I. Selverston, Abstr. Soc. Neurosci. 29, 403.16 (2003).

[13] Y. Panchin, Y. Arshavsky, T. Deliagina, L. Popova, and G. Orlovsky, J. Neurophysiol. 73, 1924 (1995).

[14] V. S. Afraimovich, N. N. Vesichev, and M. I. Rabinovich, Radiophys. Quantum Electron. 29, 795 (1986).

[15] L. M. Pecora and T. L. Carroll, Phys. Rev. Lett. 64, 821 
(1990)

[16] L. M. Pecora, T. L. Carroll, G. A. Johnson, D. J. Mar, and J. F. Heagy, Chaos 7, 520 (1997).

[17] S. Boccaletti, J. Kurths, G. Osipov, D. L. Valladares, and C. S. Zhou, Phys. Rep. 366, 1 (2002).

[18] P. Seliger, L. S. Tsimring, and M. I. Rabinovich, Phys. Rev. E 67, 011905 (2003).

[19] V. P. Zhigulin, Phys. Rev. Lett. 92, 238701 (2004).
[20] L. Glass, Nature (London) 410, 277 (2001).

[21] C. Schafer, M. G. Rosenblum, J. Kurths, and H-H Abel, Nature (London) 392, 239 (1998)

[22] R. C. Elson, A. I. Selverston, R. Huerta, N. F. Rulkov, M. I. Rabinovich, and H. D. I. Abarbanel, Phys. Rev. Lett. 81, 5692 (1998).

[23] M. Schmidt, J. Neurophysiol. 90, 3931 (2003). 